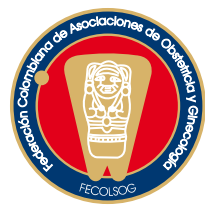

\title{
Editorial
}

\section{INEQUIDADES SOCIALES EN LA SALUD MATERNA}

«I would like for you to dream with me, to dream of a world where social justice is taken seriously».

Michael Marmot

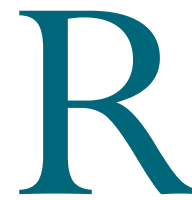

eflexionar sobre las inequidades sociales en salud como un término global asume de entrada dos grandes principios: el primero es que no se están incluyendo en la definición todas las desigualdades encontradas en salud sino sólo aquellas que adquieren el apelativo de 'inequidades', al ser desigualdades que son evitables, innecesarias e injustas. ${ }^{1}$ El segundo está relacionado con el apelativo de 'sociales', que al incluirlo de tal manera califica la inequidad como consecuencia o producto de situaciones que la sociedad puede evitar. ${ }^{2,3}$ De esta manera, el panorama de análisis se centra desde lo muy general, que serían las desigualdades en salud, hasta algo más específico como son las inequidades sociales en salud, en las que la sociedad, el tomador de decisiones y el sistema de seguridad social en salud tienen un gran nivel de responsabilidad.

Ahora bien, si se centra aún más el término hacia las inequidades sociales en la salud materna, el tema adquiere unas dimensiones de alta relevancia social pues la salud materna o mejor, la maternidad segura hacen parte fundamental de las Metas de Desarrollo del Milenio y de las políticas públicas promovidas desde la Organización Mundial de la Salud y por ende, de los respectivos ministerios. ${ }^{4-10}$

Desde 1946, la Organización Mundial de la Salud en su constitución manifestó que "los más altos estándares en salud deben alcanzarse para todos sin distinción de raza, religión, creencias políticas, condición económica o social". ${ }^{11}$ Sin embargo, se menciona que la mortalidad materna es la inequidad en salud más grande del mundo, ocurriendo el 99\% de dichas inequidades en los llamados países en vías de desarrollo. ${ }^{12}$ Esto significa que las metas propuestas desde 1946 no se han alcanzado en la salud materna y que tal vez, las distancias se han hecho más profundas y por ende, la inequidad ha aumentado entre los países.

La medicina ha hecho sus contribuciones en la salud materna al mejorar las tecnologías que están a disposición de las madres para hacer la maternidad más segura. De tal manera que la UNFPA (Fondo de Población de las Naciones Unidas) propone una estrategia de tres puntas para alcanzar la meta propuesta de reducir en un 75\% la mortalidad materna. Dichas estrategias son: 1) tener acceso a medidas de anticoncepción y embarazos no deseados; 2) tener acceso al cuidado de personal entrenado al momento del parto; 3) que la paciente con complicaciones tenga acceso oportuno al cuidado obstétrico de emergencia. ${ }^{13}$ Sin embargo, este enfoque se centra en el modelo de atención como estrategia para disminuir la mortalidad materna, sin comprender que detrás de la salud de la mujer hay un contexto social e histórico que influye de manera directa o indirecta en la ocurrencia de estos eventos no deseados en salud.

Es aquí entonces en donde se hace relevante plantear la discusión alrededor de las inequidades sociales de la salud materna y no de las inequidades sociales de la atención materna. La segunda aproximación tiene un tinte centrado en la provisión de los servicios de salud mientras que la primera tiene un énfasis en la sociedad, su entorno y su responsabilidad. Por ello, el enfoque de los determinantes sociales en salud ha sido tan acogido, aunque desde el punto de vista práctico sea más fácil para el tomador de decisiones centrarse en mejorar la provisión de los servicios de salud. 
A favor de este planteamiento estaría el hecho de que la provisión de servicios de salud para las madres en Afganistán, Bangladesh, República Democrática del Congo, Etiopía, India y Nigeria, en donde ocurren la mitad de las muertes maternas del mundo, ${ }^{12}$ es pésima, lo que significa que las inequidades mundiales en la atención en salud son muy relevantes. Asimismo, si se comparan los niveles de pobreza, inequidad de género, inequidad global, alfabetización, escolaridad y de desarrollo, las cifras de estos países están entre las peores del mundo. ${ }^{14}$

En el mundo y en los departamentos y municipios de Colombia se vigila la mortalidad materna como una entidad de salud pública de alta prioridad. Los tomadores de decisiones, los médicos e incluso las familias esperan la llegada de una intervención salvadora que disminuya sólo con su efecto y su poder todas las muertes maternas evitables que siguen ocurriendo día tras día y año tras año. Eso sería lo ideal: una solución unicausal a un problema multicausado. La realidad es que la maternidad segura se da a partir de la buena calidad en la provisión de los servicios de salud, y va más allá, detrás de las otras causas, las causas de las causas de las que habla Marmot, ${ }^{15}$ y la solución implica entonces o una intervención multimodal con muchos enfoques en diferentes niveles de jerarquía, incluyendo la atención en salud como uno de ellos, o soñando un poco, un ordenamiento real del mundo, para hacerlo más justo, con menos brechas entre ricos y pobres, con mas acceso al desarrollo para todos, con más escolaridad, menos inequidad global y menos inequidad en salud, tanto para las madres, como para la población en general.

\section{Javier Eslava-Schmalbach, M.D., M.Sc., Ph.D.}

Director, Instituto de Investigaciones Clínicas, Universidad Nacional de Colombia.

\section{REFERENCIAS}

1. Whitehead M. The concepts and principles of equity and health. Int J Health Serv 1992;22:429-45.

2. Whitehead M. What works in tackling health inequalities? Pathways, policies and practice through the lifecourse.
Sheena Asthana and Joyce Halliday. Int J Epidemiol 2007;36:259-60.

3. Whitehead M. The World Health Organisation. WHO stimulates a commitment to tackling inequalities in health. BMJ 1995;310:1472.

4. Coskun AM, Karakaya E, Yaser Y. A safe motherhood education and counselling programme in Istanbul. Eur J Contracept Reprod Health Care 2009;14:424-36.

5. Barker CE, Bird CE, Pradhan A, Shakya G. Support to the Safe Motherhood Programme in Nepal: an integrated approach. Reprod Health Matters 2007;15:81-90.

6. Madi BC, Hussein J, Hounton S, D'Ambruoso L, Achadi E, Arhinful DK. Setting priorities for safe motherhood programme evaluation: a participatory process in three developing countries. Health Policy 2007;83:94-104.

7. World Health Organization partograph in management of labour. World Health Organization Maternal Health and Safe Motherhood Programme. Lancet 1994;343:1399-404.

8. Rani R. Safe motherhood programme. Nurs J India 1993;84:231-3.

9. Dutta PK. Scope of health systems research in Child Survival and Safe Motherhood programme. Indian J Matern Child Health 1993;4:38-41.

10. Cost-effectiveness of different Safe Motherhood programme options. Safe Motherhood 1992(9 Suppl):iv.

11. WHO. Constitution of the World Health Organization. Journal [serial en línea]. 2006 [visitado 2010 Ago]; Forty-fifth edition, Supplement (October): Disponible en: http:/www.who.int/governance/eb/ who_constitution_en.pdf

12. UNFPA. Maternal Health Thematic Fund. Annual Report 2008. UNFPA; 2009 [visitado 2010 Ago]. Disponible en: http://www.unfpa.org/webdav/site/ global/shared/documents/publications/2009/mhtf_ annual_report.pdf

13. UNFPA. Safe Motherhood. UNFPA; [visitado 2010 Ago]; Disponible en: http://www.unfpa.org/public/ mothers

14. United Nations DP. Human development report 2009: Overcoming barriers: Human mobility and development. New York: Palgrave Macmillan; 2008.

15. Marmot M. Social determinants of health inequalities. Lancet 2005;365:1099-104. 